\title{
Philosophiques
}

\section{Les perspectives humaniste et politique sur les droits humains}

\section{Pablo Gilabert}

Volume 42, numéro 2, automne 2015

Dossier. Les droits humains - nouveaux développements

URI : https://id.erudit.org/iderudit/1034741ar

DOI : https://doi.org/10.7202/1034741ar

Aller au sommaire du numéro

\section{Éditeur(s)}

Société de philosophie du Québec

\section{ISSN}

0316-2923 (imprimé)

1492-1391 (numérique)

Découvrir la revue

Citer cet article

Gilabert, P. (2015). Les perspectives humaniste et politique sur les droits humains. Philosophiques, 42(2), 251-282. https://doi.org/10.7202/1034741ar

\section{Résumé de l'article}

Cet article s'intéresse au lien entre deux perspectives qui concernent la nature des droits humains. Selon la perspective " politique » ou " pratique », les droits humains sont des revendications que les individus entretiennent face à un certain nombre de structures institutionnelles, dans certains États modernes, en vertu des intérêts qui sont les leurs selon les contextes qui les mettent en jeu. Selon la perspective " humaniste » ou " naturaliste ", plus traditionnelle, les droits humains sont des revendications pré-institutionnelles que les individus entretiennent face à tous les autres individus, en vertu d'intérêts propres à l'humanité qu'ils ont tous en partage. Cet article soutient qu'une fois qu'on identifie ces deux perspectives sous leur meilleur jour, on peut voir qu'elles sont complémentaires et qu'on a en réalité besoin de chacune d'elles pour donner sens, sur le plan normatif, à la pratique contemporaine des droits humains. J’y explique comment des considérations humanistes et politiques peuvent et même doivent travailler main dans la main si on veut rendre compte du concept, du contenu et de la justification des droits humains.
Ce document est protégé par la loi sur le droit d'auteur. L’utilisation des services d'Érudit (y compris la reproduction) est assujettie à sa politique d'utilisation que vous pouvez consulter en ligne.

https://apropos.erudit.org/fr/usagers/politique-dutilisation/ 


\title{
Les perspectives humaniste et politique sur les droits humains
}

\author{
PABLO GILABERT \\ Université Concordia' \\ pablo.gilabert@concordia.ca
}

\begin{abstract}
RÉSUMÉ. - Cet article s'intéresse au lien entre deux perspectives qui concernent la nature des droits humains. Selon la perspective «politique» ou «pratique», les droits humains sont des revendications que les individus entretiennent face à un certain nombre de structures institutionnelles, dans certains États modernes, en vertu des intérêts qui sont les leurs selon les contextes qui les mettent en jeu. Selon la perspective «humaniste» ou «naturaliste», plus traditionnelle, les droits humains sont des revendications préinstitutionnelles que les individus entretiennent face à tous les autres individus, en vertu d'intérêts propres à l'humanité qu'ils ont tous en partage. Cet article soutient qu'une fois qu'on identifie ces deux perspectives sous leur meilleur jour, on peut voir qu'elles sont complémentaires et qu'on a en réalité besoin de chacune d'elles pour donner sens, sur le plan normatif, à la pratique contemporaine des droits humains. J'y explique comment des considérations humanistes et politiques peuvent et même doivent travailler main dans la main si on veut rendre compte du concept, du contenu et de la justification des droits humains.
\end{abstract}

ABSTRACT. - This essay explores the relation between two perspectives on the nature of human rights. According to the «political» or «practical» perspective, human rights are claims that individuals have against certain institutional structures, in particular modern states, in virtue of interests they have in contexts that include them. According to the more traditional «humanist» or «naturalistic» perspective, human rights are pre-institutional claims that individuals have against all other individuals in virtue of interests characteristic of their com mon humanity. This essay argues that once we identify the two perspectives in their best light, we can see that they are complementary and that in fact we need both to make good normative sense of the contemporary practice of human rights. It explains how humanist and political considerations can and should work in tandem to account for the concept, content, and justification of human rights.

1. La rédaction souhaite remercier Aude Bandini pour la traduction de cet article, d'abord publié en anglais: Gilbert, P. (2OII). Humanist and Political Perspectives on Human Rights. Political Theory, vol. 39(4), p. 439-467. (C) (20II) by SAGE Publications. Traduction autorisée avec l'aimable permission de SAGE Publications, Inc.

L'auteur souhaite remercier pour leurs commentaires très utiles, Mary Dietz, les trois évaluateurs de la revue Political Theory, ainsi que Arash Abizadeh, Elizabeth Ashford, Catherine Lu, Jesse Tomalty, et Laura Valentini.

La recherche sur laquelle est basé cet article a été financée par une subvention du Conseil de recherches en sciences humaines du Canada. 


\section{Introduction}

Cet article s'intéresse au lien entre deux perspectives différentes que l'on peut adopter sur la nature des droits humains. Selon la perspective "politique » ou encore "pratique», les droits humains consistent en un certain nombre de revendications que les individus entretiennent, face à certaines structures institutionnelles, dans certains États modernes, en vertu des intérêts qui sont les leurs selon les contextes qui les mettent en jeu. Cette perspective a été développée récemment par des spécialistes de la philosophie politique tels que Charles Beitz, Joshua Cohen et John Rawls². On l'a introduite en opposition à la perspective plus traditionnelle et dite «humaniste» ou "naturaliste ", selon laquelle les droits humains correspondent à des revendications pré-institutionnelles que les individus entretiennent face à tous les autres individus, en vertu d'intérêts propres à l'humanité qu'ils ont tous en partage $^{3}$. Cet article soutient qu'à partir du moment où l'on arrive à envisager ces deux perspectives sous leur meilleur jour, on s'aperçoit qu'elles sont complémentaires et qu'en réalité on a besoin de chacune d'elles pour donner réellement sens à la pratique des droits humains contemporaine sur le plan normatif ${ }^{4}$. La perspective humaniste met l'accent sur le fait central qu'il y a un ensemble de droits abstraits que chacun revendique contre tout autre, et ce en vertu de leur humanité commune et non de leur appartenance à une structure institutionnelle en particulier. La perspective politique, quant à elle, permet de rendre compte du fait que les droits humains sont, et devraient être mis en œuvre dans la pratique par l'identification de droits bien plus particuliers et qui sont toujours liés à des cadres institutionnels donnés. Cet article entend expliquer pourquoi il y a là deux principes qui sont en vérité compatibles et même cruciaux si on veut parvenir à rendre adéquatement compte des droits humains, et montre par conséquent dans quelle mesure leur conjugaison constitue un changement d'orientation, par contraste avec le rapport d'opposition dans lequel on conçoit d'ordinaire ces deux approches.

2. Rawls, The Laws of Peoples (Cambridge, MA, Harvard University Press, I999). Cohen, "Minimalism about Human Rights: The Most We Can Hope For? " Journal of Political Philosophy I2 (2004), I90-2I3. Beitz, "What Human Rights Mean", Daedalus I32 (2003), 36-46 (ci-dessous WHRM); "Human Rights and the Law of Peoples" in The Ethics of Assistance, D. K. Chatterjee (ed.) (Cambridge, Cambridge University Press, 2004), I93-2I4 (ci-dessous HRLP); «Human Rights " in A Companion to Contemporary Political Philosophy, $2^{\text {nd }}$ ed., R. Goodin, P. Pettit, and T. Pogge (eds), (Oxford, Blackwell, 2007), 628-637 (ci-dessous HR); The Idea of Human Rights (Oxford, Oxford University Press, 2009) (ci-dessous IHR).

3. Voir A. J. Simmons, "Human Rights and World Citizenship: The Universality of Human Rights in Kant and Locke" in Justification and Legitimacy (Cambridge, Cambridge University Press, 200I). Les partisans contemporains de l'humanisme comptent parmi eux Allen Buchanan, Simon Caney, James Griffin et Martha Nussbaum. On trouvera les références de leurs travaux dans la prochaine section.

4. Dans «Human Rights: A Freedom Centered View» (manuscrit non publié), Laura Valentini s'efforce elle aussi de proposer une combinaison de ces deux perspectives. 
Pour présenter la synthèse que je me propose de réaliser, il me semble qu'on peut identifier cinq dimensions d'analyse, et envisager cinq raisons correspondantes de défendre la thèse selon laquelle le discours des droits humains est lié à des structures institutionnelles spécifiques:

\section{Le contenu}

Les structures institutionnelles ne jouent pas seulement un rôle instrumental dans la violation ou la protection des droits humains. Elles engendrent également des relations sociales particulières, et par conséquent, des droits particuliers.

\section{La cohérence avec la pratique et la justification}

Se représenter les droits humains comme étant institutionnellement déterminés est parfaitement cohérent avec, notamment, la pratique légale et politique actuelle.

\section{L'auto-détermination et la diversité}

Partout où des institutions incarnent des formes d'auto-détermination politique, les agents peuvent identifier les droits prioritaires en fonction du contexte historique dans lequel ils se trouvent, et peuvent décider de manière autonome ce qu'ils veulent concrètement mettre en œuvre.

\section{Les devoirs corrélatifs}

Des institutions fortes permettent la plupart du temps de savoir tout de suite à qui incombe la responsabilité de faire quoi et pour qui. Voilà qui est important quand on cherche à faire en sorte que les devoirs qui répondent aux droits humains soient des devoirs parfaits plutôt que des devoirs simplement imparfaits. Même quand les devoirs sont imparfaits, il est plus approprié de considérer que c'est aux institutions plutôt qu'aux individus qu'ils incombent principalement, et cela autorise de plus une attribution des responsabilités plus souple.

\section{La possibilité de mettre en œuvre}

Avec des institutions fortes, on accroît la possibilité de respecter effectivement les droits humains, en vertu de quoi les exigences qu'ils formulent s'avèrent plus réalistes et plus pertinentes sur le plan pratique ${ }^{5}$.

Je ne doute pas un instant que de telles institutions donnent des raisons d'adopter la perspective politique. Ce qui m'intéresse ici est la question de savoir comment il faut envisager la relation entre cette perspective, si on

5. Comme j'aurai l'occasion de le montrer, les différentes questions soulevées par toute cette matrice de concepts sont en lien les unes avec les autres. Si on veut par exemple traiter de la question du contenu, il faut aussi aborder les questions des devoirs corrélatifs et de la possibilité de mise en œuvre. 
l'adopte, et la perspective humaniste. D'ordinaire, on ne présente pas vraiment l'adoption de la perspective politique comme impliquant, par principe, le rejet pur et simple de la perspective humaniste. Cependant, on l'accompagne souvent de l'idée que: I) l'humanisme a de sérieuses difficultés à rendre compte de certaines des institutions susmentionnées, et/ou que: 2) quand il s'agit de rendre compte de la nature des droits humains, on parvient à de meilleurs résultats en épousant la conception politique indépendamment de la conception humaniste. Cependant, je soutiens qu'une conception satisfaisante des institutions non seulement est compatible avec des considérations humanistes, mais même exige qu'on en tienne compte. Pour ce qui concerne le fond, je soutiendrai que la perspective humaniste identifie un ensemble de droits abstraits qui permet de sélectionner quels sont les droits particuliers adéquats que la perspective politique nous enjoint de défendre. Quant à la justification, je défends une forme d'équilibre réflexif délibératif au moyen duquel, grâce aux considérations de nature humaniste, on disposera d'une base de raisonnement substantielle. À partir de là, on pourra alors développer et renforcer notre capacité à défendre aussi bien qu'à critiquer certains aspects des droits humains tels qu'on les met actuellement en œuvre dans la pratique. Cette base est également tout à fait décisive si l'on veut expliquer pourquoi l'auto-détermination et la sensibilité à la diversité sont des choses importantes, mais aussi quelles limites il faut y poser. Pour ce qui concerne les devoirs corrélatifs, je montrerai que les droits abstraits que l'humanisme met au jour débouchent sur des exigences particulièrement précises mais néanmoins suffisamment souples pour qu'on puisse les faire correspondre à la variété des circonstances. Enfin, pour ce qui concerne la possibilité de mise en œuvre, je soutiendrai que l'humanisme permet de concevoir les devoirs dans un cadre dynamique, de manière à étendre la possibilité de mise en œuvre de certains ensembles d'actions politiques. Ce faisant, il nous sera possible de nous montrer à la fois sensible aux circonstances politiques du moment, et critique face à toute conception hâtive qui poserait ces devoirs comme fixés une fois pour toutes, ou du moins pour un temps indéfini. Par conséquent, on a absolument besoin d'une perspective humaniste pour reconnaître l'importance du rôle que jouent les institutions, encadrer la forme qu'elles prennent et les pouvoirs qui leur reviennent, et pour expliquer pourquoi il peut être nécessaire d'en créer ou de les transformer. Pour être plausible sur le plan normatif, il faut que le raisonnement politique s'élabore à partir de considérations humanistes. Il vaut mieux, une fois qu'on se les représente de la bonne manière, envisager les considérations humanistes et politiques comme fonctionnant main dans la main. On ne devrait pas développer d'approche politique indépendamment de considérations humanistes.

J'ai donné plus haut les affirmations centrales autour desquelles s'articulent les perspectives humaniste et politique. On pourrait naturellement les élaborer davantage et de manière différente. Cependant, je ne vise pas à 
défendre ici une conception précise et détaillée des droits humains. Mon objectif est plus général et porte sur un point plus élémentaire: il s'agit de montrer que (et comment) l'on devrait mobiliser simultanément les affirmations centrales qui caractérisent ces deux perspectives si l'on voulait réussir à élaborer une telle conception et qu'elle soit satisfaisante. Dans la section qui vient, j'exposerai les traits principaux de ce qui me semble être une perspective humaniste plausible. Pour ce qui est de la perspective politique en revanche, je me fonderai dans une large mesure sur la formulation qu'en a récemment proposée Charles Beitz, ceci non seulement pour économiser de l'espace, mais aussi parce que Beitz propose une défense remarquable (et peut-être les arguments les plus forts) de la conception politique. De plus, et c'est particulièrement important, Beitz défend des versions de deux propositions, les propositions (I) et (2), avec lesquelles je suis en désaccord. Il soutient ainsi que la conception naturaliste "déform[e] notre perception des droits humains à l'échelle internationale " et qu' «il vaudrait mieux envisager les droits humains sous un angle pratique, non pas comme l'application dans le champ international d'une conception philosophique qui en est indépendante, mais comme une doctrine politique qu'on a élaborée pour qu'elle joue un rôle précis dans la vie politique globale ${ }^{6}$.» Il présente son approche politique comme le «nouveau départ» qui pourrait succéder à la réfutation du présupposé traditionnel qui veut qu'on conçoive les droits humains comme des revendications que nourriraient tous les êtres humains en vertu de l'humanité qu'ils ont tous en partage?

\section{Le contenu}

Les structures politiques institutionnelles paraissent tout de suite constituer un élément central de toute conception des droits humains, car elles jouent un rôle décisif aussi bien dans la violation que dans la protection de ces droits. Les violations à grande échelle, comme le génocide nazi, sont le plus souvent le fait d'appareils d'État très organisés, lesquels sont les seules instances susceptibles de mobiliser des ressources et d'imposer des tâches à des populations entières. C'est pour les mêmes raisons que les États sont souvent les acteurs principaux de la protection des droits humains. La meilleure protection, par exemple celle de l'intégrité corporelle ou de la subsistance, est celle qui est assurée par des systèmes juridique ou de sécurité, des services sociaux et des politiques de régulation économique, tous étant à la fois équitables et efficaces. Mais ceci n'est pas suffisant pour justifier que l'on oppose perspectives politiques et perspectives humanistes. Ceci vient du fait qu'on se représente ici l'importance du rôle que jouent les institutions politiques comme étant d'ordre essentiellement instrumental. L'humaniste est sans doute tout à fait prêt à accorder qu'il nous faut porter attention aux struc-

6. IHR, 48-49. Voir aussi IO2-4.

7. IHR, 50-5I, 96. 
tures institutionnelles, attendu qu'elles servent aussi bien à violer qu'à protéger les droits humains et ce, de manière particulièrement large, tout en soutenant une conception pré-institutionnelle des droits que ces mêmes institutions sont censées permettre de violer ou de protéger.

Une opposition flagrante ne commence à se faire jour qu'à partir du moment où l'on remarque que l'existence de certaines institutions détermine le contenu des droits humains eux-mêmes. Si on prend la Déclaration Universelle des Droits de l'Homme, on peut voir que nombre des droits qui y sont mentionnés n'auraient aucun sens en l'absence de certaines institutions. Comme le soutient Beitz dans sa contestation des doctrines humanistes traditionnelles, le droit à un procès impartial, à participer au gouvernement de l'État, et à une éducation élémentaire gratuite «pose les bases de ce que serait un environnement institutionnel acceptable", et par conséquent "l'idée que de tels droits pourraient exister dans l'état de nature n'a aucun sens $»^{8}$. Voilà qui semble engendrer un dilemme pour les humanistes: soit ils rejettent leur thèse centrale, selon laquelle les droits humains doivent être ancrés dans des propriétés que tous les êtres humains partagent en tant qu'êtres humains (c'est à dire indépendamment de tout contexte institutionnel donné), soit ils courent le risque d'apporter de l'eau au moulin du scepticisme, ce qui va contre un certain nombre des exigences les plus importantes autour desquelles s'articulent les droits humains tels qu'on les met d'ordinaire en pratique'.

Ce défi que doit relever la perspective humaniste dérive de la première intuition que l'on a mentionnée dans la section précédente. Mais il est en fait possible de concevoir l'humanisme de telle sorte qu'il puisse en réalité en rendre compte. Pour le mettre en évidence, il faut faire une distinction entre les droits abstraits et les droits particuliers. En effet, on peut envisager les droits humains à plusieurs niveaux d'abstraction. On peut, d'une part, y voir un ensemble de droits particuliers qui correspondent aux revendications que les individus, dans le monde contemporain, entretiennent face à leur propre gouvernement et à leurs concitoyens, ou face à des organisations internationales, des gouvernements étrangers ou des citoyens de pays étrangers. Les droits que l'on trouve dans la Déclaration Universelle des Droits de l'Homme sont dans une large mesure de ce type ${ }^{10}$. Mais on peut aussi, d'autre part, $y$ voir un ensemble de droits abstraits portant sur des intérêts fondamentaux et partagés par tous (ou du moins par la plupart) des êtres humains, et dont la

8. WHRM, 4I ; voir aussi IHR, 55. Les droits auxquels je fais ici référence sont consignés dans les Articles IO, 2I et 26 de la Déclaration. Voir Ian Brownlie and Guy Goodwin-Hill, Basic Documents in Human Rights, 5 th ed. (Oxford, Oxford University Press, 2006).

9. À propos du problème sceptique, voir Beitz, IHR, 50, 53, 66-67, I38.

10. Cependant, certains des droits exprimés par la Déclaration sont extrêmement abstraits (par exemple, Article 3: "Tout individu a droit à la vie, à la liberté et à la sûreté de sa personne », et certains des droits qui apparaissent dans les Pactes, les traités internationaux, les constitutions ou les lois sont encore moins précis. 
protection suppose que toute personne susceptible de faire obstacle à leur satisfaction en assume la responsabilité. Par exemple, on peut dire, comme Griffin, que les droits humains protègent les conditions de l'agentivité normative humaine, lesquelles incluent un degré acceptable d'autonomie (la capacité à former et réviser sa conception de ce qu'est une vie humaine digne d'être vécue), un minimum de biens (les conditions matérielles suffisantes pour permettre aux agents de vivre comme ils l'ont choisi), et la liberté (ce qui suppose des limites à la mesure dans laquelle les agents peuvent interférer avec ce que les autres visent $)^{11}$. Ou bien, comme le fait Nickel, on peut en appeler à des intérêts fondamentaux et de nature générale, dont la protection est une condition de la vie décente. Ces intérêts d'ordre abstrait peuvent permettre de fonder la revendication de pouvoir vivre, d'être maître de sa vie, de ne pas être soumis à des traitements cruels ou dégradants, ou encore de ne pas être soumis à des traitements clairement inéquitables ${ }^{12}$. Enfin, on peut, avec Sen et Nussbaum, concevoir les droits humains comme autant de revendications visant à protéger les principales capabilités humaines, capabilités dont la satisfaction est présupposée par toute vie humainement digne, et qui par conséquent renvoient à des intérêts humains fondamentaux ${ }^{13}$. Une fois que l'on dispose d'un portrait convaincant de ce en quoi consistent à la fois ces intérêts fondamentaux et les droits abstraits qui les protègent, on peut définir des droits particuliers susceptibles d'être appliqués dans les différents contextes sociaux et politiques possibles. Des droits particuliers ainsi conçus permettront d'identifier la protection que méritent les intérêts fondamentaux dans certaines circonstances historiques.

Il y a, naturellement, des manières différentes de définir les droits abstraits. Il n'est cependant pas crucial pour ce qui nous intéresse dans cet article de répondre à la question de savoir laquelle il faudrait préférer ${ }^{14}$. Ce

11. James Griffin, On Human Rights (Oxford, Oxford University Press, 2008), 32-33.

12. James Nickel, Making Sense of Human Rights, 2nd ed. (Oxford, Blackwell, 207), 62. Pour des approches similaires, voir Allen Buchanan, Justice, Legitimacy, and Self-determination (Oxford, Oxford University Pres, 2004), I28-3 I ; Simon Caney, «Global Poverty and Human Rights: The Case for Positive Duties,» in Freedom from Poverty as a Human Right, ed. T. Pogge (Oxford, Oxford University Press, 2007), 275-302.

13. Armatya Sen, «Elements of a Theory of Human Rights ", Philosophy and Public Affairs 32 (2004), 3I 5-56; Martha Nussbaum, Frontiers of Justice (Cambridge, MA, Harvard University Press, 2006), 69-8I et 284-9I. Chez Nussbaum, cela inclut l'ensemble des conditions qui permettent de garantir les capabilités des individus, en rapport avec leur vie, leur santé et leur intégrité corporelle, l'usage de leurs sens, de leur imagination et de leur pensée, le développement de liens d'appartenance sociale, d'une relation de qualité avec les autres espèces, d'activités de jeu, et le contrôle de leur environnement politique et matériel.

14. Il serait lieu de combiner ces différentes conceptions. Griffin a tout à fait raison d'insister sur la spécificité de l'agentivité normative, quand on distingue les humains des autres êtres vivants. Mais il n'est pas nécessaire que les droits humains protègent les intérêts humains qui ne coïncideraient avec les intérêts d'aucun autre être vivant. Ce qui est crucial, c'est que les droits humains protègent ce qui est de toute première importance pour tous les humains. La différence réelle passe entre ce dont les humains ont besoin en tant que membres de groupes ou 
qu'il faut retenir pour l'instant, c'est le fait que la distinction entre des droits abstraits et des droits particuliers nous permet de montrer: I) qu'il n'y a pas d'opposition problématique entre l'approche politique et l'approche humaniste concernant le contenu des droits humains; et 2) qu'en réalité, les deux sont importantes si l'on veut rendre compte de manière adéquate de l'ensemble de ce contenu. Voilà qui s'explique par plusieurs raisons. La première est que cette distinction nous autorise à dire que chacune des deux perspectives porte sur un type de droit différent. La conception politique se concentre essentiellement sur les droits particuliers tels qu'on les trouve consignés dans la documentation juridique contemporaine, tandis que la conception humaniste porte davantage et même essentiellement sur des assertions morales, de nature plus générale. Dans la mesure où ces droits ressortent de niveaux d'abstraction différents, on ne peut pas reprocher à des affirmations qui concerneraient une de ces catégories de ne pas jouer le rôle que ne pourraient en réalité jouer que des affirmations concernant l'autre de ces catégories. Par conséquent, il n'y a peut-être pas vraiment de sens à contester la conception humaniste en disant que certains des droits de la Déclaration Universelle des Droits de l'Homme ne figurent pas dans la liste des droits abstraits qu'elle identifie. C'est exactement pour les mêmes raisons qu'il est faux de dire qu'un humaniste serait dogmatiquement contraint d'affirmer que certains des droits de la Déclaration, comme ceux que l'on a mentionnés plus tôt (droits à un procès impartial, à prendre part au gouvernement du pays, à une éducation élémentaire gratuite) ne sont pas des droits humains parce qu'ils ne sont pas valables partout, abstraction faite de toute considération sociale et politique spécifique. Cela supposerait en effet que l'humaniste confond toujours les deux catégories de droits, ce qui n'est nullement le cas.

On pourrait objecter que la distinction entre ces deux catégories de droits ne nous conduit pas très loin, parce qu'il faut, en plus, montrer qu'il $\mathrm{y}$ a de bonnes raisons de faire intervenir les deux quand on veut rendre compte de l'ensemble des droits humains. Or il ne peut y en avoir, poursuit l'objection, parce que les droits abstraits sur lesquels se concentre l'humaniste présupposent des circonstances asociales qui sont en rupture radicale avec les circonstances réelles pour lesquelles les droits de la Déclaration ont été formulés. Cependant, une telle réponse présuppose à tort une représentation atomiste de la nature humaine. Certains des premiers défenseurs de la théorie du droit naturel auraient peut être accepté une telle représentation, mais il n'y a aucune raison de penser que l'humanisme ne pourrait pas trouver de formulation telle qu'on puisse y considérer les êtres humains

de sociétés particulières, et ce dont les humains ont besoin quel que soit le contexte. Ce dernier élément pourrait bien ressembler à ce dont les autres êtres vivants ont également besoin (par exemple, pouvoir rester en vie, ou éviter une souffrance insoutenable). 
comme des êtres essentiellement sociaux ${ }^{15}$. Il faudrait alors proposer une version plus plausible et plus charitable de l'humanisme. Selon cette version, on se servirait de propriétés très générales des êtres humains, telles qu'ils les manifestent dans leur vie sociale, pour formuler des droits abstraits. Cela inclurait, par exemple, leur capacité à raisonner de manière prudentielle et morale sur ce qui est bien et juste, leur mortalité et leur vulnérabilité physique et psychologique, leur usage du langage et leur capacité relative à modifier leur environnement par des actions intentionnelles. On partirait également du principe que les êtres humains doivent faire face à une certaine rareté des ressources du point de vue matériel, qu'ils présentent certaines tendances au conflit d'intérêt social et aussi, parfois, certaines dispositions à l'agressivité et à la non-coopération, ainsi que des tendances plus bénéfiques, à la coopération, au respect, au souci de l'autre et à la sympathie. À partir de tels principes, on poserait les grandes lignes des conditions de vérité que devraient satisfaire les expressions de droits abstraits. On ne pourrait les réduire à aucune circonstance sociale particulière, mais ils demeureraient vrais dans chacune d'entre elles. L'humaniste pourrait alors encore dire que, dans tout contexte social, les individus nourrissent des prétentions abstraites au sujet des conditions de leur agentivité normative, de leur droit à ne pas être soumis à certaines formes de traitement inéquitable ou dégradant, ou à développer certaines capabilités essentielles ${ }^{16}$.

On voit donc que les humanistes peuvent très bien élaborer une représentation convaincante de ce à quoi les êtres humains "en tant que tels"

15. Voilà pourquoi je préfère le terme d' «humanisme» à l'expression «théorie naturaliste». Dans les deux cas, on se représente les droits humains, au niveau fondamental, comme des revendications morales que chaque personne nourrit face à toute autre en vertu du fait qu'elles partagent une même humanité, et on considère que ces droits ont une préséance normative sur les institutions particulières et servent éventuellement à les évaluer. Mais l'humanisme n'a pas besoin d'adopter les thèses sujettes à discussion que la plupart des partisans de la théorie du droit naturel soutiennent traditionnellement quant à ce en quoi consiste la nature humaine, ni la méthode par lesquels ils en dérivent les droits. Beitz a récemment reconnu qu'on pourrait développer une conception «naturaliste» non asociale. Mais il soutient qu'il n'en demeurerait pas moins que: I) elle serait incapable d' "intégrer ou de faire usage de considérations relatives aux fonctions discursives des droits humains au sein de la pratique existante »; 2) elle se concentrerait de manière trop exclusive sur le point de vue de ceux qui bénéficieraient de la promotion des droits humains, sans rien dire vraiment de celui ou de ceux qui devraient y contribuer ; 3) son "contenu normatif » serait «incapable de rendre compte de la liste des protections qu'on peut trouver dans la doctrine internationale des droits humains "; et 4) elle présupposerait de manière problématique qu'à chaque fois qu'il y a un décalage entre la doctrine internationale et la théorie naturaliste, cette dernière doit avoir préséance, au moins prima facie (IHR, 65-68). Je m'efforce de surmonter les objections I et 3 dans cette section, et les objections 4 et 2 dans les sections «La cohérence avec la pratique et la justification » et "Les devoirs corrélatifs".

16. Certaines caractéristiques générales de la condition humaine de ce genre soustendent la nécessité qu'il y a à se doter d'abord de structures légales. H. L. A. The Concept of Law, 2nd ed. (Oxford, Oxford University Press, I994), 9I-98 et I93-200. 
peuvent avoir droit (au sens des droits abstraits). Une approche politique pourrait tout à fait intégrer cette représentation. Beitz, par exemple, ne nie pas qu'une approche pratique pourrait avoir recours à des valeurs plus profondes, comme celles qui sont associées à l'idée de personne. Mais il craint que les idées générales de ce genre soient «trop abstraites pour permettre de régler le désaccord qui règne à propos du contenu de la doctrine des droits humains ou [...] imposent une limite arbitraire à la portée substantive de cette doctrine ${ }^{17}$ ». J'ai déjà traité du problème du scepticisme et de l'arbitraire concernant le contenu. Mais je pense également que les droits abstraits n'ont rien de trivial et qu'ils jouent un rôle tout à fait important dans l'identification des droits plus spécifiques sur lesquels la perspective politique est plus apte à se pencher. Non seulement les droits abstraits sont plausibles en l'absence du type d'institutions complexes que l'on retrouve de manière caractéristique dans les organisations modernes, mais de plus ils permettent de rendre compte de droits plus spécifiques aussitôt qu'on prend en compte les considérations factuelles qui touchent les contextes sociaux particuliers. Pour reprendre les exemples stimulants de Beitz, pourquoi faudrait-il que nous nous inquiétions des droits à bénéficier de procès impartiaux, à prendre part au gouvernement de leur pays, ou à une éducation élémentaire gratuite? Pour répondre à cette question, il nous faut non seulement faire référence à certaines propriétés du monde moderne, comme par exemple le fait qu'il existe des systèmes juridiques, que les décisions politiques se prennent dans certains cadres définis, ou encore qu'il existe des institutions éducatives formelles; il faut de plus que nous comprenions ce qui fait que certaines des manières dont ces institutions traitent les individus sont acceptables ou non. Intuitivement, on pourrait répondre correctement à cette question supplémentaire en en appelant (entre autres) au type d'intérêts et de droits abstraits dont parle l'humaniste. Il faut que les systèmes juridiques comptent de quoi mener des procès impartiaux parce que les individus ont particulièrement intérêt à ne pas être traités de manière inique. Il faut que la prise de décision politique soit ouverte à la participation des individus, car sinon leur autonomie serait gravement compromise. Et elle serait également compromise s'ils n'avaient pas le niveau d'éducation qui leur permet d'élaborer leur projet de vie de manière éclairée et critique, étant donné le contexte social qui est le leur.

Par conséquent, le discours humaniste concernant les droits abstraits conduit naturellement au discours politique concernant les droits particuliers, aussitôt qu'on prend en compte le contexte institutionnel dans lequel la protection des droits abstraits se déploie. Et le discours politique s'articule naturellement avec celui de l'humanisme dès que l'on se demande comment identifier les dispositions particulières qu'appellent les contextes institutionnels concernés. Aussi n'est-il pas nécessaire de dire, comme Beitz,

17. IHR, I 38. 
qu'au lieu d'insister sur le fait que «les droits humains se justifient sur la base de considérations d'humanité commune en tant que telle ", on devrait plutôt les voir comme des «droits spéciaux» dérivant des relations particulières que les individus entretiennent au sein du paysage institutionnel du monde moderne ${ }^{18}$. Cela supposerait en effet que les catégories de droits que l'on vient de distinguer sont radicalement hétérogènes, alors que ce n'est pas le cas. Pour prendre un autre exemple, prenons le droit, inscrit dans la Déclaration, de bénéficier d'un niveau de vie suffisant. Comme le dit Beitz, «toute explication plausible du fondement moral de ce droit devra faire référence aux relations sociales qu'entretiennent les individus, ainsi qu'à ce qui les caractérise ${ }^{19}$ ". On ne peut pas en rendre compte en considérant simplement «les propriétés que les individus pourraient être réputés posséder quand on les considère abstraction faite de la situation sociale dans laquelle ils se trouvent $\mathrm{t}^{20}$ ». Mais remarquons que le droit à un niveau de vie satisfaisant peut être envisagé soit comme un droit abstrait, soit comme un droit particulier. Quel que soit le contexte social concerné, les individus ont besoin de disposer d'un minimum de biens matériels pour subvenir à leur existence et la poursuivre dans la direction souhaitée. Dès lors, prétendre à un niveau de vie suffisant est bien, en un sens, un droit abstrait. La question de savoir de quelle forme de biens matériels les individus ont spécifiquement besoin renvoie à celle des besoins particuliers qu'ils ont selon les contextes. Pour parvenir à une liste d'exigences plus concrète à ce niveau, on peut appliquer la liste humaniste des droits abstraits à ces contextes, et ainsi élaborer la liste politique des droits particuliers.

\section{La cohérence avec la pratique et la justification}

L'un des problèmes centraux qu'identifie Beitz, à propos de l'approche humaniste traditionnelle, est qu'elle ne s'harmonise pas correctement avec la pratique des droits humains telle qu'on la trouve aujourd'hui. Elle impose alors à cette pratique un test de justification qui lui est complètement étranger. Une revendication qui se révèlerait incapable d'entrer en correspondance avec cette représentation philosophique prédéterminée - selon laquelle les droits humains dérivent de revendications que tous les humains entretiennent à l'égard les uns des autres en vertu de leur humanité commune - ne mériterait alors pas le nom de droit humain. Selon Beitz, on peut

18. WHRN, 42; voir aussi IHR, 7I-2.

19. WHRM, 42: «S'il faut juger le contenu des articles de la Déclaration ou d'autres pactes, on constate que les droits humains internationaux peuvent tout à fait de jouer un rôle dans un certain nombre de sociétés [...] celles qui présentent au moins certains signes caractéristiques de modernisation: un système légal raisonnablement bien développé [...], une économie dans laquelle une partie importante de l'emploi est occupée par l'industrie plutôt que par l'agriculture, et la capacité institutionnelle publique de faire augmenter les revenus et de fournir les biens collectifs essentiels " (43). Voir aussi IHR, 57-58.

20. HRLP, 198. 
éviter de se laisser ainsi corseter et développer plutôt une conception des droits humains qui s'élaborera à partir d'une description de la pratique internationale effective. Il dresse alors une opposition explicite entre la conception humaniste traditionnelle (qu'il nomme "orthodoxe» ou «philosophique ») et celle, politique, qui a sa préférence : la conception "pratique». Cette opposition est motivée par le fait que la conception traditionnelle est perçue comme problématiquement dogmatique. Elle laisse entendre qu'au lieu d'imposer des structures externes à la pratique des droits humains, on devrait travailler sur le plan de l'immanence, et s'efforcer de développer la «meilleure conception» possible de ce qu'elle vise à atteindre, et partir de là 21 .

Avant de chercher à savoir ce que vaut la conception pratique, il faut bien comprendre ce qu'elle recouvre. Par exemple, est-ce qu'elle requiert que l'on prenne la pratique internationale comme point de départ heuristique, dans le processus de découverte qui mènerait à la liste appropriée de droits humains? Personne ne s'en plaindrait. Les humanistes ne se plaindraient pas que nous commencions notre réflexion concernant les droits particuliers et les droits abstraits par la prise en considération de nos réactions face à ce qui se fait dans la pratique internationale. La difficulté n'est pas de savoir où il faut commencer, elle est de savoir où l'on s'en va, et pourquoi. La conception pratique, affirme-t-elle, de manière plus ambitieuse que la pratique internationale fournit-elle déjà la justification d'une liste de ce genre? Si c'était effectivement ce qu'elle affirmait, elle risquerait de glisser vers une forme manifestement problématique de conventionnalisme. Sans doute avons-nous raison de dire que ceux qui sont engagés dans la pratique des droits humains devraient croire qu'il existe certains droits humains quand et parce que les individus en ont, et non que les gens en ont quand et parce que ceux qui sont engagés dans la pratique et les mettent en œuvre croient que tel est le cas.

21. «Par contraste [avec la conception «orthodoxe» ou "philosophique»], la conception pratique se développe à partir de la doctrine et du discours des droits humains tels qu'on peut les trouver dans la pratique politique internationale. Des questions comme celles de savoir ce que sont les droits humains, quels sont les droits humains que nous pouvons avoir, ou encore celle de savoir qui a le devoir de réagir en cas de violation des droits humains, sont toutes interprétées comme faisant référence à des objets qui tombent sous la description de ce que l'on appelle «les droits humains" dans la vie internationale contemporaine, quelle que puisse être la meilleure manière de les concevoir par ailleurs. On ne présuppose en aucun cas qu'il existerait une catégorie première ou indépendante de valeurs fondamentales dont on pourrait découvrir la nature et le contenu indépendamment de toute réflexion concernant le domaine international, et qu'on pourrait alors utiliser pour interpréter et critiquer la doctrine internationale. Au contraire, on considère que le rôle fonctionnel des droits humains dans le discours et la pratique internationaux épuise l'idée de droit humain, et que le contenu de la doctrine internationale peut être défini à partir d'une réflexion concernant la meilleure manière dont cette doctrine pourrait être interprétée, à la lumière de ce rôle» (HRLP, I97; voir IHR, IO2-3). 
Si les auteurs de la Déclaration et les délégués de l'Assemblée générale des Nations Unies avaient refusé de considérer que la liberté d'expression ou la liberté de culte étaient des droits humains, nous ne serions pas convaincus d'accepter le contenu d'une telle Déclaration alternative comme correct par le simple fait que ceux qui l'ont rédigé et voté le prétendaient tel. Le simple rappel de la différence élémentaire qu'il y a entre le fait que quelque chose soit le cas, et le fait que l'on affirme ou que l'on croit que c'est le cas, n'implique aucun fondationnalisme philosophique particulièrement profond. Il s'agit seulement d'un préliminaire à toute pensée critique qui vise à identifier ce qui est vrai plutôt qu'à rapporter simplement ce que certaines personnes pensent être vrai.

Ceci étant dit, la plupart des partisans de l'approche politique ne sont pas des conventionnalistes purs et durs. Ils disent que bien que nous devrions partir des pratiques telles qu'elles sont, il se peut que nous ayons à en critiquer certains aspects, ou demandions à ce que certaines des croyances auxquelles elles donnent lieu soient révisées. Telle est certainement la position de Beitz ${ }^{22}$. À présent, comment s'y prend-on pour critiquer la pratique contemporaine des droits humains? Aussitôt que l'on se penche sur la question, on y trouve une myriade de réponses qui sont souvent opposées les unes aux autres. Les désaccords portent souvent sur la question de savoir quels sont les droits qu'il faut reconnaître, comment il faut les interpréter et les appliquer, et quels sont ceux qui doivent l'emporter sur les autres en cas de conflit. Alors que certains l'acceptent, d'autres nient qu'il y ait des droits

22. Selon Beitz, on peut raisonnablement espérer «remplacer les conceptions des droits humains qui invitent au scepticisme par une conception plus compatible avec les objectifs et la manière dont la pratique existante se déroule, sans pour autant sacrifier la possibilité de la critiquer" (IHR, I98-9). Voir WHRM, 46; HRLP, 205; IHR, I05. Voir également la critique que fait Beitz des «théories de l'accord» (IHR, chap. 4) et sa défense minutieuse des droits des femmes en cas de conflit, dans certaines cultures, quand certaines croyances morales sont dominantes (IHR, I9I04). Dans "Justice and the Priority of Politics to Morality (Journal of Political Philosophy I6 (2008), I37-64), Andrea Sangiovanni expose une conception politique nuancée, qui n'est pas une forme de conventionalisme mais une approche interprétative des institutions et qui compte trois niveaux: I) un «niveau pré-interprétatif », dans lequel on identifie l'objet commun d'interprétation; 2) un "niveau interprétatif» dans lequel on reconstruit l'intérêt et le but poursuivis par les institutions que l'on interprète et ceux des raisons pour lesquelles les agents ont pu fixer les règles, procédures et standards de ces institutions; et 3) un «niveau post-interprétatif» dans lequel, sur un mode explicitement critique, on étend la compréhension interprétative des institutions et on en dérive un ensemble de premiers principes, afin de les réguler. Cette conception pose au moins un problème, qui est qu'on ne voit pas bien en quoi (3) est suffisamment différent de (2), de sorte qu'on puisse disposer de quoi fonder la critique des standards qu'on a identifiés au niveau (2). Si ce à quoi on aboutit au niveau (3) est plus qu'une simple description des principaux présupposés sur lesquels reposent les raisons identifiées en (2), par exemple un ensemble d'éléments de justification au moyen desquels on pourrait évaluer ces raisons, il faut encore expliquer d'où viennent ces éléments de justification. 
humains de nature socioéconomique ${ }^{23}$. Alors que certains l'acceptent, d'autres nient qu'il y ait un droit humain à la démocratie ${ }^{24}$. Alors que certains l'acceptent, d'autres nient que les droits civils et politiques aient une priorité sur les droits socioéconomiques ${ }^{25}$. Comment sommes-nous censés mettre un terme à de telles disputes? Beitz propose que nous nous prononcions de manière critique sur ces désaccords en fonction de la manière dont les différentes positions cadrent avec les «objectifs" ou le «rôle» que la pratique des droits humains déjà existante entend atteindre ou remplir ${ }^{26}$. Mais comme il le reconnaît également ${ }^{27}$, les objectifs et le rôle que le discours des droits humains entend atteindre ou jouer ne sont pas moins matière à débat que le contenu de la Déclaration lui-même. En effet, alors que certains l'affirment, d'autres nient que son rôle se réduise à identifier les conditions sous lesquelles on peut autoriser que des interventions étrangères interfèrent dans la vie interne des Éétats ${ }^{28}$. Alors que certains l'affirment, d'autres nient que les droits humains fondent des responsabilités incombant aux individus, et que ces responsabilités soient aussi importantes que celles qui incombent aux institutions (entre autres aux États) ${ }^{29}$. Tandis que certains l'affirment, d'autres nient que la pratique des droits humains ne soit pour l'essentiel qu'un mécanisme voilé au moyen duquel certains Éétats plus puissants rationalisent la domination qu'ils exercent sur les Éétats plus faibles ${ }^{30}$. Com-

23. Voir les articles contenus dans Pogge, Freedom from Poverty as a Human Right; ainsi que Maurice Cranston, What Are Human Rights? (London, Bodley Head, I973).

24. Carol Gould, Globalizing Democracy and Human Rights (Cambridge, Cambridge University Press, 2004); Joshua Cohen «Is There a Human Right to Democracy? " in The Egalitarian Conscience, C. Sypnowich (ed.) (Oxford, Oxford University Press, 2006), 226-48.

25. Voir la discussion générale dans Amartya Sen, Development as Freedom (New York: Anchor Books, I999).

26. IHR, I05-6.

27. IHR, I08.

28. Rawls, The Law of Peoples; James Nickel, "Are Human Rights Mainly Implemented by Intervention?) in Rawls's Law of Peoples. A Realistic Utopia? R. Martin and D. Reidy (ed.) (Oxford, Blackwell, 2006), 263-77.

29. Henry Shue, "Mediating Duties", Ethics 98 (1988), 687-704; Thomas Pogge, World Poverty and Human Rights, 2nd ed. (Cambridge: Polity, 2008).

30. Ces discussions animaient déjà les rédacteurs de la Déclaration. Voir Mary Glendon, A World Made New: Eleanor Roosevelt and the Universal Declaration of Human Rights (New York, Random House, 200I). On peut trouver un exemple touchant à la question de la responsabilité dans l'insistance avec laquelle René Cassin affirmait le caractère primordial des devoirs des individus (comme ceux des institutions en dessous et au dessus de l'État), à l'égal des devoirs incombant aux États (93, II3-4). C'est la raison pour laquelle Cassin proposa, juste avant son adoption, que le titre de la Déclaration soit changé, de "Déclaration internationale des droits de l'homme» en "Déclaration universelle des droits de l'homme». Glendon explique: "[Cassin...] expliqua par la suite que le titre "universelle" voulait dire que la Déclaration engageait moralement chacun, et pas seulement les gouvernements qui avaient voté pour son adoption. La Déclaration universelle [...] n'était pas un texte "international" ou "intergouvernemental"; il s'adressait à l'humanité toute entière, et se fondait sur une conception unifiée de l'être humain" (I6I). Le dernier paragraphe du Préambule de la Déclaration universelle confirme cette idée, 
ment peut-on identifier et défendre les critères de ce qui serait la "meilleure conception » de la pratique des droits humains? Il se peut qu'il ne soit pas suffisant d'exiger, sur le plan méthodologique, qu'une telle conception entre en cohérence avec une description de la pratique en question, car tout individu qui prend part à cette pratique pourrait soutenir que la doctrine des droits humains devrait refléter sa propre conception (elle-même sujette à controverse) de ce que vise cette pratique. De plus, la simple idée que la justification de certaines affirmations dépendrait de leur cohérence avec la pratique qui leur donne jour est en soi une affirmation philosophiquement discutable, et qu'il faudrait défendre sur le plan philosophique (ce qui nous mènerait sur le type de terrain que les partisans de la perspective politique préfèreraient sans doute éviter).

\section{Amender la conception pratique}

Je n'affirme pas qu'il faudrait purement et simplement rejeter la conception "pratique» que propose Beitz. Je défends plutôt l'idée qu'il faudrait l'amender, et qu'il serait important de tenir compte des considérations humanistes si on veut par la suite l'améliorer. Pour ce qui est du premier point, suivons Beitz lorsqu'il propose de faire une distinction entre le concept des droits humains et ce qui concerne leur contenu et leur justification ${ }^{31}$. L'idée est que l'on pourrait éviter les désaccords en proposant d'abord une description de ce qu'il faut comprendre par ce concept (quels sont les objectifs et le rôle des droits humains) tel qu'il est utilisé dans la pratique contemporaine, puis en proposant des arguments solides concernant son contenu et sa justification. Ainsi, selon Beitz, la signification des droits humains est qu'ils incarnent « les normes constitutives d'une pratique globale dont le but est de protéger les individus contre les menaces qui découlent des actes et des manquements de leurs gouvernements ${ }^{32}$ ». Avec ce concept en mains, nous pouvons poursuivre et proposer un aperçu détaillé du contenu et de la justification des droits humains en identifiant: I) quels sont les intérêts individuels primordiaux qui sont fréquemment menacés dans les États modernes; 2) quelles sont les "exigences primaires" auxquelles les gouvernements doivent satisfaire afin que les intérêts de leurs citoyens soient protégés;

spécifiant que celle-ci présente «l'idéal commun à atteindre par tous les peuples et toutes les nations afin que tous les individus et tous les organes de la société [...] s'efforcent, par l'enseignement et l'éducation, de développer le respect de ces droits et libertés et d'en assurer, par des mesures progressives d'ordre national et international, la reconnaissance et l'application universelles et effectives ». Voir également la Convention sur l'élimination de toutes les formes de discrimination à l'égard des femmes, qui vise à être appliquée "dans les domaines politique, économique, social, culturel et civil ou dans tout autre domaine» (Article I).

31. IHR, IO-II, I04-I06, I26. Bien entendu, la manière dont on conçoit la signification a des conséquences sur la manière dont on conçoit le contenu et la justification. Beitz en a conscience, mais à ses yeux, la relation va aussi dans le sens inverse.

32. IHR, I 97. 
3) quelles sont les raisons "secondaires" pour lesquelles, à première vue, des gouvernements ou agents étrangers devraient se porter au secours ou bien dénoncer les gouvernements qui ne satisferaient pas à leurs obligations premières ${ }^{33}$.

Il faut remarquer que, dans une telle conception, la manière dont on conçoit le concept de droits humains détermine de manière assez stricte la manière dont on en conçoit à la fois le contenu et la justification. Par exemple, dans la définition que l'on a donnée aux droits humains, les États jouent un rôle de premier ordre. Dès lors, toute conception du contenu et de la justification des devoirs correspondant aux droits humains que l'on pourra proposer devra elle aussi considérer les États comme acteurs principaux. Or ceci implique qu'on rassemble dans une même définition plusieurs affirmations importantes (par exemple que les droits humains ne sont pas d'essence institutionnelle et étatique, ou qu'il y a des devoirs positifs fondamentaux $^{34}$ ) dont bien des acteurs, dans la pratique, contestent la validité. Comme nous l'avons vu, les débats qui entourent actuellement ces questions vont très loin. On pourrait réagir en modifiant la description de la signification de la pratique, de manière à la rendre moins sujette à controverse. Mais le problème vient peut-être du fait qu'en réalité on ne peut pas si facilement séparer la conception de la signification des considérations relatives au contenu et à la justification. Plutôt que de procéder par étapes, en partant de la signification pour ensuite s'intéresser au contenu et à la justification, nous aurions peut-être intérêt à traiter les trois ensemble, au sein d'une tentative qui s'efforcerait toujours de parvenir à un «équilibre réfléchi » dans la manière dont nous pensons les droits humains. Il nous arrivera sans doute de sentir qu'il est nécessaire de réviser notre conception de la signification qu'il faut reconnaître à la pratique, lorsque celle-ci entrera en conflit avec ce que nous aurons jugé être le contenu et la justification de certains des droits qui y sont mis en jeu. D'autre part, il ne faut pas oublier qu'un tel équilibre est le but de la délibération, et non de la description: ce qui nous intéresse avant tout, ce n'est pas de découvrir ce que nous (ou d'autres) avons pu penser jusque là concernant les droits humains, mais bien ce que nous devrions en penser ${ }^{35}$.

\section{Intégrer les considérations humanistes}

L'ensemble de ces suggestions (en ce qui concerne l'équilibre réfléchi et sa nature délibérative) déboucherait sur une conception pratique révisée que j'adopterais volontiers. Dans cette conception révisée, les considérations humanistes quant à ce que les êtres humains, en tant que personnes, peuvent

33. IHR, I09, I37.

34. IHR, I09, II4-I 5, I28-I 29.

35. Sur la distinction entre les conceptions descriptive et délibérative de l'équilibre réfléchi, voir T. M. Scanlon, «Rawls on Justification » in The Cambridge Companion to Rawls, S. Freeman (dir.) (Cambridge, Cambridge University Press, 2003), I39-67. 
exiger de tous les autres, jouent un rôle crucial dans la manière dont on se représente la pratique, c'est-à-dire sa signification, ses contenus et sa justification ${ }^{36}$. Par conséquent, comme je l'ai mentionné plus haut, l'idée de «commencer» par la pratique internationale des droits humains paraît tout à fait raisonnable. Il est absolument vrai que nous ne nous représenterons pas clairement l'ensemble des droits humains particuliers qu'il serait justifié d'adopter tant que nous n'aurons pas pris en compte les contextes particuliers dans lesquels les discussions et les revendications concernant les «droits humains" interviennent. Si on fait abstraction de ces contextes, la liste des droits abstraits à laquelle on parviendra, quelle qu'elle soit, sera beaucoup trop vague et inapte à guider l'action politique à la fois nationale et internationale. Mais à partir du moment où on envisage les choses dans la perspective de l' «équilibre délibératif réfléchi » que je propose, il s'avère qu'il y a en réalité plus de raisons de s'attacher à la perspective humaniste que de s'en passer. Il y a deux idées que je voudrais défendre à ce sujet. Ces deux idées s'articulent à celles que j'ai avancées dans la section précédente (laquelle montrait qu'il était tout à fait concevable d'envisager l'humanisme comme étant compatible avec, et même comme nécessitant, la prise en compte du contexte particulier, si l'on voulait identifier et justifier les droits particuliers qui permettent l'application des considérations morales beaucoup plus générales qui entrent en jeu au niveau des droits abstraits). J'aurai, dans les trois sections suivantes, l'occasion de me prononcer sur la question cruciale de savoir s'il faut faire intervenir la notion d'État dès le niveau de la définition.

Le premier point est que les considérations humanistes interviennent déjà au sein de la pratique contemporaine des droits humains. Cela paraît évident à la lecture du Préambule et des Articles I et 2 de la Déclaration. Le Préambule s'ouvre en faisant référence à la «reconnaissance de la dignité inhérente à tous les membres de la famille humaine et de leurs droits égaux et inaliénables », laquelle constitue "le fondement de la liberté, de la justice et de la paix dans le monde». Le fait que nous devrions «reconnaître» la dignité des êtres humains ainsi que leurs droits est incompatible avec l'idée que c'est la Déclaration elle-même qui serait tout simplement à l'origine de leur création ${ }^{37}$. L'Article premier affirme: que «Tous les êtres humains naissent libres et égaux en dignité et en droits" et "sont doués de raison et de conscience et doivent agir les uns envers les autres dans un esprit de fraternité », tandis que l'Article 2 soutient: que «Cchacun peut se prévaloir de

36. Même dans une perspective épistémologique cohérentiste, certains principes moraux substantiels peuvent entrer en fonction de manière relativement invariante. Voir Geoffrey Sayre-McCord, "Coherentist Epistemology and Moral Theory " in Moral Knowledge, W. Sinnot-Armstrong and M. Timmons (eds), (New York, Oxford University Press, I996), I37-89, I 5 I.

37. Comme le soutient Glendon (op. cit., I76), cela rend la Déclaration incompatible avec une lecture juspositiviste. La Déclaration reconnaît les droits; elle ne les confère pas. 
tous les droits et de toutes les libertés proclamés dans la présente Déclaration, sans distinction aucune, notamment de race, de couleur, de sexe, de langue, de religion, d'opinion politique ou de toute autre opinion, d'origine nationale ou sociale, de fortune, de naissance ou de toute autre situation.. » Ces clauses partent clairement du principe qu'il y a des propriétés attachées aux êtres humains en tant que tels (par exemple le fait qu'ils soient "doués de raison et de conscience»), et que les droits humains sont compatibles avec le respect de la «dignité » de chaque personne, abstraction faite de ce qui les distingue (comme leur « race, $[(\ldots])$ couleur, $[(\ldots])$ sexe, $[(\ldots])$ langue, $[(\ldots])$ religion, $[(\ldots])$ opinion politique ou $[(\ldots])$ toute autre opinion, $[(\ldots])$ origine nationale ou sociale, $[(\ldots])$ fortune, $[(\ldots])$ naissance ou $[(\ldots])$ toute autre situation.»). Ces affirmations imposent un cadre humaniste à l'ensemble de la Déclaration. Elles nous obligent à garder à l'esprit que, lorsque nous cherchons à identifier quelles sont les revendications particulières que la période contemporaine met en jeu (par exemple les droits socioéconomiques particuliers dont la Déclaration fait mention par la suite, et qui sont manifestement liés à l'existence de systèmes économiques à l'âge de la modernité), c'est à ce que méritent les humains conçus tels que mentionné plus haut que nous pensons. Toutes ces affirmations paraissent intuitivement plausibles. Elles ne sont ni moralement ineptes, ni philosophiquement triviales. De fait, les rédacteurs de la Déclaration se sont livrés à des débats houleux à ce sujet. Prenant conscience du fait qu'ils ne pouvaient pas, ni n'avaient en réalité besoin de, se mettre d'accord sur la question de savoir si certains droits trouvaient leur fondement dans une conception religieuse, dans une théorie métaphysique ou morale, sous-jacente en particulier ou non, ils se sont finalement accordés sur le fait qu'il y avait un désaccord portant sur les fondements des droits humains. Mais il est tout aussi vrai que certaines conceptions morales et philosophiques moins fondamentales faisaient l'objet de discussions entre eux. Parmi elles se trouvaient les conceptions associées aux notions de "fraternité ", de "dignité », "conscience ", "liberté ", "égalité », et la manière dont il fallait concevoir les positions relatives des individus, groupes sociaux et États ${ }^{38}$.

38. Voir Glendon, $o p$. cit. Les rédacteurs de la Déclaration consultèrent un comité de l'UNESCO comprenant des penseurs issus d'une très grande variété de traditions. Ils conclurent qu'un accord était possible sur certaines convictions essentielles et sur la base desquelles on pourrait établir la Déclaration, mais qu'il ne serait pas sage de tenter d'aller plus loin, et de déterminer ensemble les doctrines religieuses, morales ou philosophiques plus fondamentales dont on pourrait le mieux les dériver $(77-78$, II 2, 222, 226). Il faut remarquer que cette conclusion ne veut pas dire que les idées ou les valeurs philosophiques n'ont pas d'importance pour le débat politique international sur les droits humains, mais que celles-ci n'ont pas besoin d'être d'un niveau aussi fondamental que celles qui interviennent inévitablement dans la réflexion philosophique (au sein de cultures, de religion ou de théories morales spécifiques). Bien qu'ils aient laissé la question des principes ultimes de côté, les rédacteurs ont néanmoins indubitablement débattu et adopté des conceptions morales substantielles (38-42; 68-69; 75-76, I4 I-42, I46-47), dont des considérations relatives à l'humanité commune des indivi- 
Le second point concerne la question du raisonnement public global pour ce qui a trait à la signification, au contenu et à la justification des droits humains. L'idée que je soutiens ici est que des considérations humanistes peuvent nous aider à en concevoir à la fois la procédure et la substance. Comme l'ont soutenu certains partisans de la perspective politique, les droits humains sont devenus monnaie courante dans les débats contemporains concernant la justice globale, ou du moins ses exigences les plus pressantes. Or ces débats ne prennent pas la forme de séminaires de philosophie. Chaque participant n'y est pas invité à chercher ce qui pourrait constituer le fondement ultime des droits humains. Le but (du moins, pour la plupart d'entre eux) est de parvenir à un consensus qui fournisse une base commune à partir de laquelle on pourra mener l'action politique internationale. Il est alors important d'éviter ce qu'on pourrait considérer comme étant les connotations fondationnalistes caractéristiques des versions philosophiques traditionnelles de l'approche humaniste. Dès lors, l'approche politique a sans doute raison de pencher pour ce que Cohen appelle un «minimalisme de la justification », qui consiste à mettre entre parenthèses le débat concernant les fondements philosophiques ultimes ${ }^{39}$. Cependant, il faut aussi éviter les raccourcis stratégiques. Le but n'est pas seulement de parvenir au consensus, mais de l'établir sur des bases méthodologiques et des hypothèses valides sur le plan normatif, sans quoi ce consensus ne sera qu'un modus vivendi dépourvu de stabilité et d'autorité morale. Bien des partisans de la conception politique en ont parfaitement conscience, et considèrent que le raisonnement public global ne consiste certainement pas simplement à rendre compte des points sur lesquels des croyances normatives préexistantes entrent en conflit, ni à fournir aux forums internationaux de quoi alimenter la manipulation et les menaces purement rhétoriques ${ }^{40}$. Mais la question demeure de savoir comment nous pouvons identifier et défendre une proposition concernant les procédures et les objectifs qui seraient appropriés en matière de raisonnement public. Devrions-nous le faire en nous abstenant d'avoir recours à toute idée du type de celles que défend l'humanisme?

Il me semble que la réponse est non. Comme on l'a vu précédemment, le débat international qui entoure la question des droits humains regorge d'affirmations morales générales relatives au statut des individus dans leurs relations sociales les uns avec les autres, et ces affirmations ne sauraient être rattachées et réduites exclusivement à un cadre institutionnel existant, puisqu'elles sont justement censées permettre l'évaluation de tout cadre de

dus, leurs intérêts fondamentaux, et leur dignité au sein de l'existence sociale de manière générale $(227-33)$.

39. "Minimalism About Human Rights», I92.

40. Cohen, Ibid.; Kenneth Baynes, "Discours Ethics and the Political Conception of Human Rights », Ethics \& Global Politics 2 (2009): I-2 I. 
ce genre. Des considérations humanistes interviennent d'ores et déjà dans la discussion qui, au sein de la pratique internationale, entoure la question des droits humains, et il semble, à la réflexion, que ce soit de manière légitime. En effet, une discussion de ce type (du moins telle que l'envisage la plupart de ceux qui y participent) vise non seulement à améliorer les structures institutionnelles existantes, mais aussi à les faire évoluer progressivement. Notre dignité en tant qu'agents doués de "raison et de conscience » et notre devoir d'agir dans un "esprit de fraternité » contribuent à former les raisons morales qu'on peut avoir de se joindre aux discussions internationales qui se tiennent au sujet des droits humains particuliers qu'il faudrait reconnaître et de la manière dont il faudrait les mettre en œuvre. Il est important que l'on dispose d'une forme de discussion qui, si elle vise à aboutir à un consensus, ne soit pas de nature purement stratégique et fasse la part belle au respect car, entre autres choses, on se doute que la dignité d'autrui serait violée si on lui imposait des institutions qu'il n'aurait pas eu le choix d'accepter de manière autonome. C'est pour la même raison qu'il est important que la discussion publique globale soit inclusive, que tous les discours pertinents puissent s'y faire entendre, ce qui implique non seulement les représentants gouvernementaux, mais aussi les individus et les organismes de la société civile, qu'ils soient nationaux ou internationaux.

On comprend alors que l'idéal du minimalisme de la justification trouve ses racines dans des considérations d'ordre à la fois normatif et empirique. Le principe empirique est celui selon lequel il existe dans le monde moderne une diversité culturelle si large qu'il est très peu probable que le raisonnement public ne parviendra jamais à des réponses convergentes, concernant la question des fondements philosophiques ultimes. Le principe normatif est celui selon lequel les structures politiques devraient se soumettre aux décisions auxquelles ceux qu'elles sont susceptibles d'affecter ont donné leur consentement éclairé. Il est raisonnable d'avoir ainsi recours à des pratiques de justification publique robustes, mais relativement «minimales », précisément parce notre respect pour l'autonomie d'autrui requiert que nous justifiions les institutions que nous partageons sur la base de prémisses morales qu'il soit susceptible d'accepter. Mais dans le même temps, il ne faut pas perdre de vue le fait qu'on ne peut pas s'attendre à ce qu'une telle justification entraîne une convergence d'opinions sur des prémisses morales plus profondes encore, alors qu'elles sont bien trop discutables pour que tout le monde les reconnaisse comme valables (au moins sur le court terme).

Comme on l'a vu dans la section précédente, l'engagement en faveur de l'humanisme peut aussi contribuer à donner forme au contenu des droits particuliers que nous pouvons venir à reconnaître. On peut effectivement dire de la plupart des droits humains de la Déclaration qu'ils sont, pour reprendre Beitz, « des critères appropriés aux institutions des sociétés modernes ou en modernisation, coexistant au sein d'une économie politique globale dans laquelle les êtres humains sont amenés à faire face à un ensemble de 
menaces prédictibles ${ }^{41}$ ». Mais pour que nous puissions reconnaître certaines menaces comme normativement pertinentes et susceptibles de donner lieu à des revendications en termes de droits humains, il nous faut partir du principe qu'il y a certains intérêts humains moraux importants que ces menaces sont susceptibles d'affecter. Or l'humanisme intervient dans le raisonnement public global comme un moyen de mettre au jour une compréhension commune de ce que pourraient être à la fois ces intérêts et les droits abstraits qui les protègent. Par conséquent, quand nous disons que le raisonnement public global doit rechercher les fondements sur lesquels évaluer les structures institutionnelles nationales et internationales, cela veut aussi dire que cette évaluation doit prendre en considération la manière dont les différents ordonnancements de ces structures peuvent affecter certains intérêts importants que tous les êtres humains ont en partage. Si on faisait l'économie de cette strate substantielle dans le raisonnement, le contenu du débat public global perdrait de vue une part importante de ce qui le dirige, ainsi qu'une grande partie de ce qui fait son intérêt sur le plan normatif.

\section{Auto-détermination et diversité}

Un autre élément qui peut motiver l'adoption d'une perspective politique sur les droits humains est le fait que celle-ci est particulièrement bien armée quand il s'agit de reconnaître l'importance de l'auto-détermination sur le plan national et celle de la diversité culturelle. Lorsqu'on a affaire à des institutions qui incarnent des formes d'auto-détermination politique, les agents peuvent d'une part identifier les types de droits dont la mise en œuvre est prioritaire étant donné les circonstances historiques dans lesquelles ils s'inscrivent, et d'autre part décider de manière autonome quelles implémentations particulières il faudrait entreprendre. La souveraineté nationale et la diversité ont toujours constitué des sujets importants dans le débat sur les droits humains. Même entre les rédacteurs de la Déclaration, il y eut des débats enflammés, certains d'entre eux craignant qu'on se serve du discours des droits humains pour remettre en question l'indépendance des nations ou leur culture $\mathrm{4}^{42}$. On ne peut nier que la perspective humaniste impose des contraintes sévères à la souveraineté des États, ainsi qu'à tout argument qui prétendrait se fonder sur des traditions culturelles. Mais la perspective politique accorderait que l'un des intérêts des droits humains consiste précisément à poser des limites à ce que l'on peut faire au nom de la souveraineté nationale et de l'identité culturelle. Par conséquent, il n'y a pas réellement d'opposition entre les conceptions politique et humaniste sur ce point.

Cependant, il se pourrait qu'il y ait une divergence quant à ce sur quoi on met l'accent. La conception humaniste ne tient pas pour acquise l'orga-

41. WHRM, 44. Voir aussi IHR, 58.

42. Glendon, op. cit., chap. I 2. 
nisation actuelle du monde en États-nations ou en peuples ${ }^{43}$. Il importe avant tout que les individus soient en mesure de déterminer la forme des processus politiques qui ont des répercussions sur leurs intérêts fondamentaux, et qu'ils choisissent le type de mise en œuvre des droits humains le plus approprié étant donné leur culture et la mesure dans laquelle ils y sont attachés (ou du moins les aspects de cette culture qui n'entrent pas en conflit avec les droits humains). Le statut des gouvernements et des États est largement dérivé à partir de ce point. La question de savoir s'il faut les conserver tels qu'ils sont dépend dans une large mesure de ce qu'ils font pour les individus et leurs droits fondamentaux.

Par conséquent, l'approche humaniste s'avère particulièrement utile dans la mesure où elle nous apporte la distance critique dont nous avons besoin à l'égard des institutions politiques et des cadres culturels. Grâce à cette distance critique, nous pouvons nous apercevoir qu'il y a des interactions positives et des interactions négatives entre, d'une part, l'engagement humaniste à l'égard des droits abstraits, et, d'autre part, l'exigence de respecter la diversité culturelle et les institutions incarnant une forme d'auto-détermination politique. Les interactions positives sont essentiellement de trois ordres, chacun d'entre eux servant à montrer que les perspectives politique et humaniste peuvent et même devraient marcher main dans la main. Au niveau épistémique, il faut naturellement remarquer qu'on peut agencer les droits abstraits de différentes manières dans différents contextes culturels, et que la présence de mécanismes de responsabilisation politique permet aux agents concernés d'identifier les mises en œuvre qui leur paraissent appropriées au travers de droits, d'institutions et de politiques particulières. Des mécanismes de ce genre donneront aussi aux agents un moyen de décider à quels droits donner la priorité si leur mise en application donnait lieu à des conflits. Un second niveau d'interaction est de nature motivationnelle. La volonté de mettre en place et de conserver des institutions et des politiques correspondant à la mise en

43. Voilà qui s'oppose particulièrement à la conception que défend Rawls dans The Laws of Peoples, où l'auteur considère que ce sont les peuples plutôt que les individus qui constituent les unités fondamentales de l'analyse normative internationale, et où il réduit la liste des droits humains établie par la Déclaration de manière à ce qu'elle s'aligne sur les préférences de certains cadres culturels. Un humaniste pourrait accorder, sur le plan pragmatique, qu'il faille renoncer à imposer certaines revendications de droits par le biais d'interventions étrangères. Mais il n'en conclurait pas pour autant qu'il ne s'agit pas de droits humains, ni qu'on ne devrait pas en faire des préoccupations centrales dans le raisonnement public global. Les autres perspectives politiques sont moins restrictives. Pour Cohen, ce sont les individus et non les peuples qui constituent les unités normatives de base, et, selon lui, la raison publique globale traite les droits humains comme des «normes associées à l'idée d'appartenance ou d'inclusion au sein d'une société politique organisée ", ce qui laisse ouverte la question de savoir si la société politique en question est d'essence nationale ou globale ("Minimalism About Human Rights", I97). Voir aussi Beitz, "Social and Cosmopolitan Liberalism ", International Affairs 75 (1999), 515-29. 
œuvre des droits humains se voit renforcée lorsque celles-ci ne sont pas imposées de manière paternaliste, mais imputables à ceux qu'elles touchent. Ce type de motivation est également renforcé si on associe le discours des droits humains à des histoires particulières concernant des expériences vécues par certaines communautés ou certains groupes. Les Allemands ont tiré de la réflexion qui a suivi le traumatisme de la période nazie, et de la réévaluation de la tradition de l'Aufklärung, des ressources particulièrement riches pour consolider l'engagement en faveur des droits humains. On trouve quelque chose de similaire en Amérique latine, avec une réflexion critique sur les relations complexes qu'ont entretenues les colonialistes européens, les immigrants et les populations autochtones, ou par l'intermédiaire d'une autre réflexion, portant sur les coûts imposés par les différentes dictatures militaires des années I970 et I980, en termes de droits civils, politique et sociaux. Enfin, l'auto-détermination politique et la capacité à se rapporter à ses propres communautés culturelles sont des éléments qui ont de la valeur en eux-mêmes. La première donne une plus grande marge d'expression à l'autonomie des individus, et la seconde sert leurs intérêts en leur permettant de développer des liens fraternels forts les uns avec les autres.

À tous ces égards, la perspective humaniste et la perspective politique se complètent. En réalité, elles sont importantes pour se limiter l'une l'autre. L'approche humaniste risque en effet de retomber dans une forme de théorie fondationnaliste dont on ne voit pas bien comment elle pourrait permettre d'orienter l'action politique dans des contextes sociaux particuliers. Quant à l'approche politique, elle risque de tenir trop de choses pour acquises sur la base d'un simple statu quo, et, ce faisant, elle pourrait échouer à rendre compte des aspirations morales universalistes qui sont pourtant décisives si on veut que la pratique des droits humains se développe progressivement dans la politique moderne. L'accent que la perspective politique met sur les structures politiques particulières donne à l'humanisme un moyen d'éviter un "universalisme abstrait» qui ne rendrait pas compte du fait que les droits humains sont des droits que l'on élabore et que l'on cherche à atteindre de différentes manières dans différents contextes, et qu'il n'est pas indifférent sur le plan moral, motivationnel et épistémique (en plus, cela va de soi, du plan prudentiel) que de telles élaborations et de telles quêtes soient entreprises par des agents politiquement responsabilisés ou non. La conception humaniste, pour sa part, permet de maintenir une distance critique à l'égard de toute configuration politique ou culturelle existante, en nous rappelant que leur statut normatif dépend toujours de leur cohérence avec le projet général qui est celui d'assurer à chaque individu les conditions d'une existence digne. Lorsque les dispositifs politiques et culturels existants deviennent des entraves plutôt que des moyens de réaliser ce projet (quand, sur le plan national ou international, ils conduisent à piétiner ou à ne pas tenir compte des inté- 
rêts fondamentaux des individus), l'humanisme nous aide à conserver une vision claire de la direction dans laquelle les réformes politiques doivent se faire. Par conséquent, il n'y a rien de surprenant à ce que le discours des droits humains ait exercé une influence majeure sur les propositions visant à limiter la souveraineté nationale en renforçant les institutions infranationales et supranationales, dans le but de renforcer la capacité de ceux qui sont affectés par les actions des États de défendre leurs intérêts fondamentaux ${ }^{44}$. Du point de vue des droits humains, il n'y a rien de sacro-saint dans cette invention de la modernité que sont les États-nations. Lorsqu'il y a une corrélation négative entre la promotion des intérêts humains et la souveraineté nationale ou l'identité culturelle, la conception humaniste nous donne une vision suffisamment claire de là où se trouvent les priorités.

\section{Les devoirs corrélatifs}

Dire qu'un individu (le titulaire du droit) a le droit ou peut prétendre à un certain objet $\mathrm{O}$ implique que les autres agents (ceux à qui incombe l'obligation) ont un certain nombre de devoirs corrélatifs. Ces devoirs peuvent être négatifs, comme le devoir de ne pas priver le titulaire du droit d'avoir $\mathrm{O}$, ou positifs, comme le devoir de fournir $\mathrm{O}$ au titulaire du droit, ou de protéger son accès à $\mathrm{O}$ contre autrui. En règle générale, il est assez facile de déterminer en quoi consistent les devoirs négatifs, parce que dans la plupart des cas ils imposent l'obligation de s'abstenir. Pour ce qui est des devoirs positifs en revanche, les choses sont beaucoup plus compliquées. C'est notamment le cas avec les droits socioéconomiques. La Déclaration en nomme plusieurs (comme le droit à bénéficier d'un niveau de vie suffisant, d'une éducation ou d'un travail), mais elle ne précise pas vraiment à qui incombent les devoirs corrélatifs, et quelles sont les actions qu'ils devraient réaliser pour respecter effectivement ces droits. Certes, cette indétermination est délibérée et appropriée dans une certaine mesure. Les rédacteurs voulaient que la Déclaration puisse s'appliquer dans différents contextes, aussi bien dans le présent que dans le futur, et que chaque pays ait suffisamment de latitude pour choisir la meilleure manière de mettre ces droits en œuvre. Par exemple, le caractère très général de la formulation des droits socioéconomiques visait à permettre qu'il y ait plusieurs manières possibles de concevoir les contributions respectives des mécanismes du marché, de l'État et des contribuables volontaires, selon ce qui serait le plus susceptible de fonctionner compte tenu de l'histoire et des traditions des différents pays. La Déclaration, et plus encore le Pacte international relatif aux droits économiques, sociaux et culturels, tient ainsi compte de la limitation des ressources des pays pauvres, et présente ainsi l'obligation de respecter les droits socioéconomiques comme

44. David Held, Global Covenant (Cambridge: Polity, 2004). 
étant de nature progressive et impliquant l'intervention d'une coopération internationale ${ }^{45}$. Ceci a conduit certains critiques à reprocher à ces droits humains de n'être pas des droits réels, mais de simples objectifs qu'il serait désirable d'atteindre. Leurs partisans ont alors répondu que ces objectifs étaient obligatoires, et qu'il existait des mécanismes institutionnels capables de le resserrer de telle sorte qu'on puisse identifier, au fur et à mesure de leur processus de mise en œuvre, les cibles précises, les types d'agents concernés et les types d'actions à mettre en place pour y parvenir ${ }^{46}$.

Imaginons que cette réponse aux critiques soit correcte, et que l'on puisse effectivement se représenter les droits socioéconomiques comme posant, outre un certain nombre de devoirs parfaits, un ensemble d'objectifs prioritaires qu'on peut se permettre d'atteindre de manière graduelle plutôt que forcément immédiate et entière, et en sollicitant l'intervention de différents agents. Une telle réponse aurait aussi l'avantage de désamorcer la tentation que l'on pourrait autrement avoir d'adopter une vision politique particulièrement étroite dans laquelle un droit humain ne peut exister que s'il y a aussi des agents institutionnels à qui peuvent incomber, et qui peuvent s'acquitter, des devoirs parfaits corrélatifs qui y correspondent et s'ils peuvent ce faisant mettre en œuvre ce droit, immédiatement et entièrement. On peut cependant aussi remarquer qu'il peut y avoir un autre problème, même si on adopte une vision politique moins étroite. La solution au problème dont on vient de parler semble en effet soulever un nouveau problème pour toute conception humaniste qui repose sur l'idée philosophique que les droits humains sont une espèce de droits moraux ${ }^{47}$. Cette nouvelle difficulté, telle que la formule Beitz, vient du fait que d'ordinaire on considère que les droits moraux constituent la base de devoirs parfaits, devoirs qui obligent certains individus en particulier à réaliser les actions particulières qui mettent immédiatement et entièrement en œuvre le droit dont il s'agit. Or, comme on vient de le voir, il ne semble pas que ce modèle soit respecté dans le cas des droits socioéconomiques tels que les conçoivent la Déclaration universelle et le Pacte international. Dans ces documents, en effet, on se concentre tout d'abord essentiellement sur les devoirs qui incombent aux institutions, les responsabilités incombant aux individus semblent alors être largement secondaires (par exemple celle de veiller sur la bonne marche des institutions concernées). Ensuite, ces documents sont assez ouverts quant à la question de savoir quelles sont les institutions qui doivent être impliquées en particulier, et dans quelle mesure leurs actions mettront en œuvre les droits en

45. Voir le Préambule et les Articles 22 et 28 de la Déclaration, ainsi que les Articles 2.I, II.I, I3.2, I4 et 23 du Pacte.

46. Pour une formulation classique de la critique, voir Cranston, What Are Human Rights? Pour voir comment les droits et les objectifs peuvent être reliés, voir Nickel, Making Sense of Human Rights, 24-26, 8I.

47. Beitz, HR, 63 I. 
question. Dès lors, concevoir les droits humains comme des revendications de type moral pourrait conduire à se demander combien des droits établis par la Déclaration, comme les droits socioéconomiques, sont réellement des droits. Une vision politique plus large ne donnerait pas prise à un scepticisme de ce genre, puisqu'on n'y part pas du principe que les droits qu'on trouve dans la pratique internationale des droits humains ont à rentrer dans un moule philosophique prédéterminé. On pourrait avoir l'impression que toutes ces réflexions donnent des raisons de croire qu'il vaudrait mieux éviter les considérations humanistes et développer une perspective politique qui en serait totalement indépendante.

Mais ce n'est pas le cas en réalité. Il y a quatre points importants à mentionner ici. Premièrement, il faut se rappeler que l'humanisme n'est pas obligé de rejeter l'idée d'une distribution institutionnelle des responsabilités. Il ne présuppose en aucune manière que ceux à qui la mise en œuvre immédiate des droits incombe devraient, quel que soit le moment, être forcément des individus. L'idée essentielle ici est que les individus sont bien ceux à qui, fondamentalement, incombe la mise en œuvre des droits, mais ils ne sont pas forcément ceux à qui elle incombe le plus immédiatement. Ils ont une obligation collective de protéger les intérêts humains fondamentaux, obligation qui est indépendante de toutes les institutions existantes, et qui perdurerait même en leur absence. Les institutions sont des créations humaines, dont l'un des rôles devrait être de s'acquitter de cette obligation. Une fois mises en place, les institutions pourraient très bien devenir l'entité principale chargée de la satisfaction immédiate de cette obligation ${ }^{48}$. Puisqu'il semble très probable qu'un gouvernement soit capable d'assurer que le peuple qui se trouve sous sa juridiction ait accès à de la nourriture durant une période de crise économique, pourquoi irions-nous donc soutenir que ce serait plutôt aux individus d'agir par euxmêmes? Dans ce cas, ceux qui souffrent de la faim devraient adresser leurs revendications, en premier lieu, au gouvernement. Mais ceci ne vient pas du fait que le gouvernement serait celui à qui incombe fondamentalement la mise en œuvre de tous les droits. Cela vient plutôt du fait que le gouvernement est souvent celui qui offre au peuple les moyens les plus équitables et les plus efficaces d'assurer la mise en œuvre du droit à la subsistance de ceux qui ont faim.

Un second point concerne la nature des droits et devoirs moraux. L'humaniste n'a aucune raison d'adopter une théorie morale dans laquelle rien n'est un droit à moins qu'il existe des devoirs corrélatifs qui soient parfaits. On

48. Ce ne sont pas les seuls à qui les droits incombent de manière immédiate. Si Harry git dans la rue et qu'il meurt à moins qu'on ne lui porte assistance dans les plus brefs délais, Mary ne pourra pas dire qu'elle n'est pas autorisée à délivrer les premiers soins avant l'arrivée de l'ambulance, parce que le seul devoir du système de soins officiel est de porter assistance aux gens comme Harry. L'obligation qui incombe à Mary découle des droits humains de Harry. En outre, dans la mesure où il arrive parfois que les institutions fonctionnent très mal et qu'une aide urgente soit nécessaire, les gens ont parfois raison d'agir sans attendre que les institutions n'interviennent ou ne soient réformées. 
peut tout à fait considérer que les droits moraux fondent différents types de devoirs, dont certains sont parfaits et d'autres imparfaits. Prenons le droit moral à la vie. Ce droit fonde certainement un devoir parfait de ne pas tuer (tout en acceptant l'idée qu'il puisse y avoir des exceptions, comme par exemple le droit de tuer en cas de légitime défense). Mais on peut tout à fait dire, sur le plan moral, qu'il faut sauver les gens chaque fois qu'ils se trouvent dans des circonstances où leur vie est menacée. Tous les autres ont, prima facie, une très bonne raison d'agir pour se porter à l'aide de ceux qui se trouvent dans de telles circonstances, du moins s'ils peuvent le faire sans qu'il leur en coûte de manière déraisonnable. Mais on ne peut pas à l'avance savoir qui, s'il devait y en avoir un, s'avèrera avoir le devoir parfait d'effectuer un sauvetage. Il faut examiner la situation, et savoir qui peut agir, à quel prix, pour décider de la question de savoir si un devoir parfait incombe ou non à quelqu'un. Or, en cherchant à le déterminer, nous tenons pour acquis que la situation d'extrême nécessité dans laquelle se trouve la victime crée une bonne raison, pour tous ceux qui sont en mesure d'agir, de considérer très sérieusement de le faire effectivement. Ce devoir imparfait, plus large, est tout à fait censé, comme le sont les objectifs généraux que visent les obligations corrélatives des droits humains socioéconomiques des individus dans les pays en développement. Il y a une certaine indétermination concernant l'identification des individus à qui incombent les devoirs et celle des actes répondant aux devoirs positifs imparfaits qui sont corrélatifs de la plupart des droits. Mais cette indétermination se retrouve à tous les niveaux du raisonnement normatif. Alors dans la mesure où nous pouvons raisonnablement concevoir aussi bien des devoirs parfaits que des devoirs imparfaits à titre de devoirs corrélatifs des droits, au niveau de la théorie morale comme au niveau de la théorie politique, le problème que semblait poser le décalage entre la conception morale et la conception politique des droits humains n'a plus de raison de se poser.

Un troisième point, lié au précédent, est le fait que le passage des devoirs imparfaits aux devoirs parfaits, et des mécanismes d'assistance interpersonnels aux mécanismes d'assistance institutionnels ne présente pas de véritable solution de continuité. L'humaniste y aurait naturellement recours. Ici encore, l'approche politique et l'approche humaniste devraient, si on les conçoit de la bonne manière, marcher main dans la main. Dans la mesure où, en temps normal, les cadres institutionnels, parce qu'ils attribuent clairement des tâches précises à effectuer à ceux à qui incombe la mise en œuvre des droits, constituent l'instance la plus susceptible de fournir des ressources à la fois efficaces et équitables, l'humaniste aura recours à une organisation politique des droits concernés. C'est parce qu'il y a des raisons pour que tous les individus voient leurs besoins satisfaits et qu'on ne leur demande pas de faire plus que leur juste part quand il s'agit de porter assistance à autrui que les institutions s'avèrent être l'instance appropriée pour la mise en œuvre des droits. Par conséquent, quand ces institutions sont déficientes ou qu'elles manquent, les individus ont une obligation pro tanto de les réformer ou de 
les créer. Et quand elles existent et qu'elles fonctionnent bien, ils ont l'obligation de les maintenir dans cet état. Voilà comment on peut concevoir une division du travail moral, qui exprime plutôt qu'il ne se substitue au type de considérations morales que tout ensemble d'individus impliqués dans la vie sociale trouverait sans doute acceptables ${ }^{49}$. La conception politique peut accepter cette idée. Elle n'a pas besoin de s'accompagner d'un culte fétichiste des institutions, selon lequel les droits humains n'existent que là où il existe, et parce qu'il existe, des institutions qui en organisent la mise en œuvre.

En dernier lieu, la perspective humaniste offre une autre contribution positive, en lien avec ses implications pratiques dans l'attribution des responsabilités quand il s'agit de mettre les droits humains en œuvre. On dit communément que quand il est question de la protection des droits humains d'un individu, c'est au gouvernement sous lequel vit cet individu qu'incombe en première instance l'obligation de respecter ces droits. Un gouvernement peut cependant manquer à ses devoirs, notamment celui de faire ce qui est nécessaire. Dans de telles circonstances, on dit souvent que les autres gouvernements ont des responsabilités de "deuxième niveau ", celles de condamner le premier gouvernement (s'il choisit de ne pas apporter d'aide alors qu'il le pourrait) ou bien de l'assister (s'il veut mais ne peut pas fournir ce qui serait nécessaire). Beitz adopte cette conception dans son modèle «à deux niveaux» des responsabilités associées aux droits humains ${ }^{50}$.

Dans une perspective humaniste, cette conception à deux niveaux de l'assignation des responsabilités est peut-être trop faible. Peut-être vaudraitil mieux dire que tous les gouvernements devraient travailler main dans la main plutôt que successivement, s'ils veulent favoriser l'instauration de conditions dans lesquelles les droits humains de tous les êtres humains seront protégés. Prenons l'important Article 28 de la Déclaration, avec le cadre qu'il pose: "Toute personne a droit à ce que règne, sur le plan social et sur le plan international, un ordre tel que les droits et libertés énoncés dans la présente Déclaration puissent y trouver plein effet.» On peut interpréter cet article de différentes façons. Une approche humaniste serait particulièrement intéressante. Si les droits humains sont en premier lieu des revendications que chaque personne peut entretenir et opposer à toute autre, alors il faut soumettre les structures nationales et internationales à un test qui permette de savoir si elles réussissent effectivement à protéger les droits humains de chaque individu. Si certaines formes d'actions internationales simultanées sont plus rapides, ou si elles impliquent une répartition de la charge de la contribution plus large ou plus égalitaire, alors il n'est pas besoin de considérer que les instances de l'étranger n'ont que des devoirs de « deuxième niveau ». Imaginons par exemple qu'une grande pauvreté sévisse

49. Pablo Gilabert, "Contractualism and Poverty Relief ", Social Theory and Pratice 33 (2007), 277-310.

50. IHR, IO9. 
dans le pays $\mathrm{A}$, tandis qu'une grande prospérité règne dans le pays $\mathrm{B}$, et que le gouvernement de A puisse parvenir à éradiquer la pauvreté dans $\mathrm{A}$, mais qu'il doive pour cela faire disparaître la classe moyenne de A et affaiblir la marge de négociation de A dans les relations commerciales qu'il entretient avec B. Dans de telles circonstances, ne serait-il pas juste que B intervienne en offrant une aide ou en contribuant à organiser le marché, de sorte que les inégalités de moyens de négociation n'aient pas pour effet que le moins bien doté voie ses opportunités économiques drastiquement diminuées? Cela n'aurait rien d'une obligation si nous nous en tenions à la conception «à deux niveaux ", mais ce pourrait être le cas si nous pensons que les institutions, nationales comme internationales, doivent (entre autres) jouer le rôle d'instruments grâce auxquels les individus du monde entier, conformément à l'esprit de fraternité ou de solidarité, veillent sur les droits humains de tous $^{51}$.

\section{La possibilité de mise en œuvre}

Intéressons-nous à présent à la dernière raison qui milite pour l'adoption de la conception politique, et qui touche aux conditions de mise en œuvre des droits. Être quelque chose d'effectivement faisable est décisif pour toute conception normative, y compris une conception qui serait centrée sur les droits humains. Une conception normative doit être telle que non seulement elle doit nous aider à critiquer certains dispositifs sociaux en montrant qu'ils sont loin d'incarner l'idéal, mais en plus de cette dimension évaluative, elle doit permettre de fonder des prescriptions d'orientation générale, indiquant comment on pourrait passer des dispositifs critiquables à de meilleurs dispositifs. Ceci nécessite cependant que l'on prenne en compte les éléments relatifs à la possibilité de mettre les droits en œuvre, car ils identifient les limites de nos capacités à changer les choses. Plus précisément, une conception normative des droits humains doit non seulement identifier un ensemble de droits putatifs R, tels que (I) leur mise en œuvre soit moralement désirable; il faut en outre que les droits que rassemble R soient tels que (2) leur mise en œuvre soit effectivement possible, depuis là où nous en sommes actuellement, et qu'elle perdure une fois qu'elle aura été effectuée. Enfin, ces considérations de désirabilité et de possibilité de mise en œuvre se conjuguent pour aboutir à l'exigence que $(3)$ le processus de réforme qui conduit à la

51. Des considérations du même ordre s'appliquent aux droits qui sont moins directement reliés aux droits moraux de tous contre tous - comme certains droits civils et politiques qui encadrent la relation des gouvernements avec leurs citoyens. Comme on l'a vu dans la section intitulée "Le contenu ", on peut voir de tels droits comme la particularisation de droits abstraits plus fondamentaux et plus généraux. Étant donné cette idée, ainsi qu’un engagement humaniste en faveur de la solidarité universelle, on peut conjuguer les droits relatifs aux agents visant à améliorer le respect des droits de ses propres concitoyens aux devoirs indifférents aux agents de favoriser les droits des citoyens de n'importe quel environnement politique. 
mise en œuvre effective de $\mathrm{R}$ doit pouvoir être accompli sans que le prix à payer pour cela, pour ceux qui seront concernés, ne soit déraisonnable ${ }^{52}$.

Dès lors, la perspective politique est particulièrement adéquate, puisqu'en se concentrant sur les dispositifs économiques, institutionnels et culturels particuliers, elle peut rendre compte des clauses (2) et (3), en plus de la clause (I). Par contraste, la position humaniste pourrait paraître indifférente au contexte politique, et par conséquent se limiter à poser des objectifs désirables abstraits, sans du tout rendre compte de la question de savoir si et comment on pourrait les atteindre, à un prix acceptable pour ceux qui sont concernés.

Je voudrais cependant mentionner deux points pour défendre l'idée que même pour ce qui concerne la question de la possibilité de mise en œuvre, on ne devrait pas développer la perspective politique indépendamment d'une perspective humaniste. Premièrement, souvenons-nous qu'on peut concevoir les revendications relatives aux droits humains selon différents niveaux de généralité. On peut ainsi distinguer les droits abstraits, les droits inscrits dans la Déclaration, les droits qui sont établis par les traités et pactes internationaux, et les constitutions nationales, ainsi que les revendications que certains individus peuvent nourrir à l'endroit des gouvernements, ou d'autres organisations et individus, selon les circonstances. Cette liste procède par généralité décroissante, puisque l'on passe des énoncés moraux abstraits à leur expression et leur mise en œuvre précise. Les conditions (I) à (3) s'appliquent à chaque niveau, mais la sensibilité au contexte sur laquelle l'approche politique insiste devient de plus en plus pertinente à mesure que l'on passe du premier niveau au second, puis au troisième et dernier. Cependant, on n'a pas de raison d'éviter la perspective humaniste ici. Au contraire, il y a des raisons de la convoquer. Comme on l'a vu dans la dernière section, l'existence d'institutions solides garantira et facilitera grandement la mise en œuvre équitable des droits humains. Quand elles existent, bien des problèmes d'action collective peuvent être résolus. Dès lors, la mise en ouvre des droits humains peut être assurée de manière plus équitable et plus efficace. Et c'est sans doute là une excellente raison de vouloir adopter la perspective politique. Mais pour d'autres raisons que l'on a déjà mentionnées, l'humanisme bien compris recommanderait également que l'on porte attention aux éléments de contexte qui nous permettront de concevoir la meilleure expression et la meilleure mise en œuvre des droits abstraits possible, en fonction des circonstances historiques particulières dans lesquelles on se trouve. Il ne faut pas non plus négliger le fait que l'humanisme nous aiderait à poser le cadre dans lequel effectuer cette tâche. L'accessibilité à un régime de droits humains et sa stabilité seront accrues si on mobilise les raisons humanistes que les gens peuvent avoir de les vouloir.

52. Pablo Gilabert, "Global Justice and Poverty Relief in Nonideal Circumstances", Social Theory and Practice 34 (2008): 4I I-38. 
Et pour évaluer les coûts qu'engendrerait la transition, on aura également besoin de prendre ces raisons en compte.

Deuxièmement, il faut remarquer que non seulement on peut concevoir les droits humains à différents degrés de généralité, mais ceux-ci recouvrent également une certaine complexité sur le plan temporel. En effet, les dispositifs culturels et institutionnels présents peuvent faire figure d'obstacles à la mise en œuvre des droits humains, mais ces obstacles pourraient disparaître ou être surmontés à l'avenir. Ainsi, certains États puissants ont entravé la reconnaissance des droits humains comme ayant une force exécutoire. Jusqu'à aujourd'hui, les États-Unis n'ont pas ratifié le Pacte international sur les droits économiques, sociaux et culturels. Les pays pauvres, pour leur part, sont souvent dans l'incapacité de mettre pleinement en œuvre les droits de leur peuple en raison de défaillances institutionnelles ou de manque de ressources. Et nous sommes encore bien loin de pouvoir assurer une culture solide de solidarité cosmopolite, et l'existence d'institutions internationales qui seraient capables de coordonner et mettre en œuvre les efforts visant à garantir les droits humains de chacun. Cela pourrait avoir pour résultat de rendre impossible la pleine réalisation des droits de la Déclaration à court terme. Mais à long terme, ces obstacles pourraient être surmontés. Il n'est pas question de caviarder la liste des droits établis par la Déclaration, simplement parce que, par exemple, il n'est pas possible de les mettre pleinement et immédiatement en œuvre à un prix raisonnable. Dans la mesure où leur mise en œuvre est compatible avec les réalités de l'existence humaine dans la vie sociale moderne, il faut les garder en tête à titre de directives à suivre pour le changement culturel et institutionnel. La pratique des droits humains est toujours sous-développée sur le plan politique. Il s'agit, comme le formule Beitz avec justesse, d'une "pratique émergente ${ }^{53}$ ». Et il ne fait aucun doute que les conceptions politique et humaniste devraient travailler main dans la main pour que cette pratique se développe. Mais il est important de se garder de penser que les éléments qui constituent le contexte actuel sont fixés pour toujours, alors qu'ils sont précisément l'objet de l'évaluation critique et la cible de la réforme historique. Il y a un grand danger à tenir pour constant ce qu'on devrait plutôt reconnaître comme variable. Par exemple, en ces temps de mondialisation, il serait sans doute trop restrictif de ne se concentrer que sur les États comme agents de la justice globale. La recherche d'un équilibre délibératif réfléchi dans laquelle nous sommes engagés nous conduit à adopter une vision plus large. Celaci ne vient pas seulement du fait que, de fait, les États perdent de leur capacité d'agir sous l'influence de mécanismes structurels globaux qui échappent à leur contrôle. Cela découle aussi du fait que, sur le plan normatif, l'émergence d'agences non gouvernementales

53. IHR, 42-44. Voir la page I2 I sur la question de l'expansion de ce qu'il a été possible de mettre en œuvre dans l'histoire. 
et supranationales pourrait bien mettre en évidence qu'il est désirable que la protection des droits humains soit davantage favorisée. La perspective humaniste est particulièrement utile si on veut défendre cette thèse, car elle fournit précisément le type de distance critique à l'égard du statu quo dont on a besoin pour pouvoir concevoir comment repousser les limites de ce qui est politiquement faisable à l'échelle du temps historique ${ }^{54}$.

Pour conclure, remarquons qu'il ne faut pas se désespérer du fait qu'on ne puisse pas mettre certains droits complètement et immédiatement en œuvre pour l'instant. Au contraire, les obstacles culturels et institutionnels qui existent à présent devraient nous encourager à prendre conscience du fait que les droits fonctionnent à des niveaux différents de généralité, si bien que certaines catégories de droits peuvent sans doute être mises en œuvre de différentes manières et dans différentes mesures, en fonction des contextes. Plus encore, il faut reconnaitre qu'il existe des devoirs dynamiques, ceux de créer les conditions culturelles et institutionnelles qui permettront un jour une mise en œuvre des droits qui n'est pas encore possible actuellement ${ }^{55}$. Ces deux idées peuvent tout à fait être intégrées à la conception politique, tant que celle-ci n'abaisse pas prématurément son niveau normatif afin qu'il s'accorde avec les propriétés contingentes et éphémères du statu quo. L'approche humaniste fournit un correctif constant et nécessaire contre la tentation d'une adaptation normative de ce type, en nous aidant à garder un œil sur ce dont les êtres humains, en tant qu'êtres humains, ont besoin, et ce à quoi ils peuvent aspirer pour s'assurer une vie digne.

\section{(Traduit de l'anglais par Aude Bandini)}

54. Nous pouvons, avec Rawls, considérer une conception des droits humains comme une "utopie réaliste », dont les exigences sont à la fois désirables et possibles à mettre en œuvre, et remarquer qu'il n'est pas facile d'établir les limites de ce qui est "possible en pratique », car celles-ci «ne sont pas données par ce qui existe actuellement, car nous pouvons, dans une plus ou moins grande mesure, faire changer les institutions sociales et politiques, et bien d'autres choses encore" (Law of Peoples, 5-7, I2). Cependant, la théorie de Rawls elle-même est incapable de déterminer dans quelle mesure nous pouvons effectivement faire changer nos institutions ainsi que la culture qui les sous-tend. Nous devrions également reconsidérer la recommandation émise par Beitz, en faveur d'une conception de la pratique des droits humains centrée sur l'État, et être sensible à la mise en garde concernant le fait que nous "tenons certains faits relatifs à la structure politique du monde comme fixes, et envisageons l'objectif de la pratique des droits humains au sein de cette structure» (IHR, I28). Non seulement certains de nos buts normatifs doivent évoluer quand certains faits évoluent, mais il se peut aussi que nous devions changer certains faits pour qu'ils correspondent à nos buts normatifs existants, quand ils sont les bons.

55. Pablo Gilabert, "The Feasibility of Basic Socioeconomic Human Rights: A Conceptual Exploration", Philosophical Quaterly 59 (2009): 659-8I. 